hep-ph/0207157

IFIC/02-28

UWThPh-2002-20

\title{
Ruling out four-neutrino oscillation interpretations of the LSND anomaly?
}

\author{
M. Maltoni ${ }^{a}$, T. Schwetz ${ }^{b}$, M. A. Tórtola ${ }^{a}$ and J. W. F. Valle ${ }^{a}$ \\ ${ }^{a}$ Instituto de Física Corpuscular - C.S.I.C./Universitat de València \\ Edificio Institutos de Paterna, Apt 22085, E-46071 Valencia, Spain \\ ${ }^{b}$ Institut für Theoretische Physik, Universität Wien \\ Boltzmanngasse 5, A-1090 Wien, Austria
}

\begin{abstract}
Prompted by recent solar and atmospheric data, we re-analyze the four-neutrino description of current global neutrino oscillation data, including the LSND evidence for oscillations. The higher degree of rejection for non-active solar and atmospheric oscillation solutions implied by the SNO neutral current result as well as by the latest 1489-day Super-K atmospheric neutrino data allows us to rule out $(2+2)$ oscillation schemes proposed to reconcile LSND with the rest of current neutrino oscillation data. Using an improved goodness of fit (g.o.f.) method especially sensitive to the combination of data sets we obtain a g.o.f. of only $1.6 \times 10^{-6}$ for $(2+2)$ schemes. Further, we re-evaluate the status of $(3+1)$ oscillations using two different analyses of the LSND data sample. We find that also $(3+1)$ schemes are strongly disfavoured by the data. Depending on the LSND analysis we obtain a g.o.f. of $5.6 \times 10^{-3}$ or $7.6 \times 10^{-5}$. This leads to the conclusion that all four-neutrino descriptions of the LSND anomaly, both in $(2+2)$ as well as $(3+1)$ realizations, are highly disfavoured. Our analysis brings the LSND hint to a more puzzling status.
\end{abstract}

Key words: neutrino oscillations, sterile neutrino, four-neutrino models

PACS: 14.60.P, 14.60.S, 96.40.T, 26.65, 96.60.J, 24.60

Email addresses: maltoni@ific.uv.es (M. Maltoni), schwetz@thp.univie.ac.at (T. Schwetz), mariam@ific.uv.es (M. A. Tórtola), valle@ific.uv.es (J. W. F. Valle). 

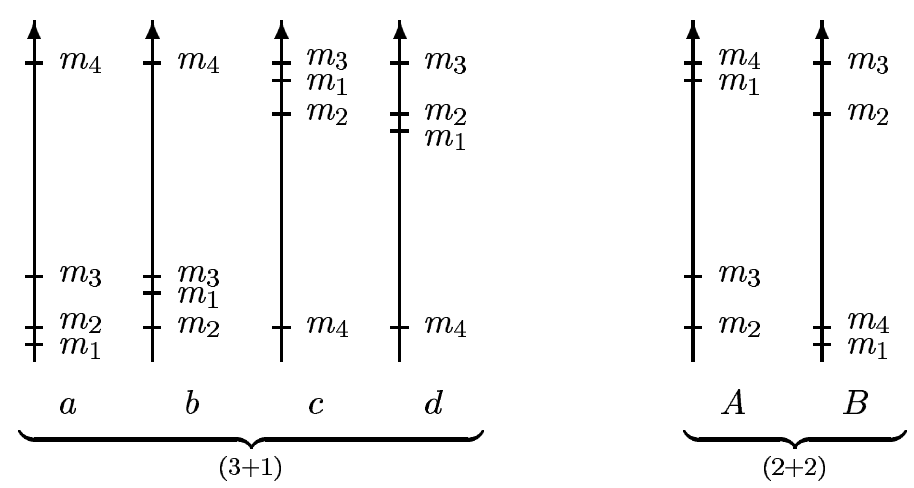

Figure 1. The six types of four-neutrino mass spectra. The spacings in the vertical axes correspond to the different scales of mass-squared differences required in solar, atmospheric and short baseline neutrino oscillations.

\section{Introduction}

The atmospheric neutrino data [1, 2, 3], including the most recent 1489 Super$\mathrm{K}$ data sample provide strong evidence for $\nu_{\mu}$ oscillations into an active neutrino (mainly $\nu_{\tau}$ ) with $\Delta m_{\mathrm{ATM}}^{2} \sim 2 \times 10^{-3} \mathrm{eV}^{2}$ [4]. On the other hand, apart from confirming, once again, the long-standing solar neutrino problem [5, 6, 7, 8, 9], the recent results from the Sudbury Neutrino Observatory (SNO) [10 have given strong evidence that solar neutrinos convert mainly to an active neutrino flavor. This suggests that an extension of the Standard Model of particle physics is necessary in the lepton sector, capable of incorporating the required $\nu_{e}$ conversion. Although certainly not yet unique [11, 12], the most popular explanation of the solar neutrino data is provided by the active large mixing angle (LMA) neutrino oscillation hypothesis, characterized by a neutrino mass-squared difference $\Delta m_{\mathrm{SOL}}^{2} \lesssim 10^{-4} \mathrm{eV}^{2} \| 4$.

In contrast, reactor and accelerator neutrino data [13, 14, 15, 16, 17] lead to negative results. However, the LSND experiment [18, 19] has provided positive results, which may or may not be confirmed by the forth-coming MiniBooNE experiment [20]. The required neutrino mass-squared difference $\Delta m_{\mathrm{LSND}}^{2} \gtrsim$ $0.2 \mathrm{eV}^{2}$ is in conflict with the neutrino mass-squared differences indicated by solar and atmospheric data in a minimal three-neutrino picture. Four-neutrino models [21, 22, 23, 24, 25, 26, 27] potentially account for all current oscillation data. The status of four-neutrino descriptions has been presented in Ref. 28. An exhaustive list of four-neutrino references can be found in Ref. [29].

The six possible four-neutrino mass spectra are shown in Fig. 1. For the case $\Delta m_{\mathrm{LSND}}^{2} \gg \Delta m_{\mathrm{ATM}}^{2}, \Delta m_{\mathrm{SOL}}^{2}$, which we tacitly assume in order to reconcile the LSND anomaly with solar and atmospheric data, these schemes can naturally be divided into two very different classes, usually called $(3+1)$ and $(2+2)$ mass schemes [30]. It is important to note that $(3+1)$ mass spectra include the threeactive neutrino scenario as limiting case. In this case solar and atmospheric 
neutrino oscillations are explained by active neutrino oscillations, with masssquared differences $\Delta m_{\mathrm{SOL}}^{2}$ and $\Delta m_{\mathrm{ATM}}^{2}$, and the fourth neutrino state gets completely decoupled. We will refer to such limiting scenario as $(3+0)$. In contrast, the $(2+2)$ spectrum is intrinsically different, as there must be a significant contribution of the sterile neutrino either in solar or in atmospheric neutrino oscillations or in both.

In recent studies [28] it has been realized that there is considerably tension in four-neutrino fits to the global data. In such global four-neutrino analyses one is faced with the problem that there are different data sets, which all give very good fits if analyzed separately. Problems arise due to the combination of the different data sets in a four-neutrino framework. In this letter we reevaluate the status of four-neutrino interpretations of the LSND anomaly in the light of the recent solar [0, 7, 10] as well as atmospheric [2] neutrino results. To evaluate the quality of the fit we will apply appropriate statistical methods, which are especially sensitive to the combination of different data sets in a global analysis. We find that both the SNO NC result as well as the 1489-day Super-K atmospheric neutrino data strongly reject against sterile neutrino conversions. This essentially rules out $(2+2)$ mass schemes. We also re-evaluate the status of $(3+1)$ schemes by considering two different analyses of LSND data [19, 31]. We will further elaborate the result of previous studies [30, 32, 33, 34, 35, 36, 37] that in $(3+1)$ schemes the LSND signal is in serious disagreement with bounds from short-baseline (SBL) experiments reporting no evidence for oscillations [13, 14, 17]. The net result is that all four-neutrino descriptions of the LSND anomaly, both in $(2+2)$ as well as $(3+1)$ realizations, are strongly disfavoured by the data. This brings the LSND anomaly to a theoretically more puzzling status. We note that also cosmology put strong constraints on four-neutrino schemes (for recent analyses see Ref. [38]).

The plan of the paper is as follows. In Sec. 2 we briefly describe our parameterization and approximations used in the global four-neutrino analysis. In Secs. 3 and 4 we summarize the solar and atmospheric neutrino data and their analysis [4. In Sec. 5 we describe the SBL data we are using, and we compare the two different analyses of the LSND data [19, 31]. In Sec. 6 we show that $(2+2)$ oscillation schemes are ruled out because of the tension between solar and atmospheric data, whereas in Sec. 7 we present our re-analysis of the disagreement in SBL data in $(3+1)$ oscillation schemes in light of the two LSND samples. In Sec. 8 we show that the goodness of fit is very bad in all four-neutrino cases. Furthermore, we compare the relative quality of the fit for the schemes $(3+1)$ and $(2+2)$, as well as the three active neutrino case $(3+0)$. The quantitative statistical criteria to analyze the conflict between different data sets, which are used in Secs. 6, 7 and 8, are formulated in the appendix A. In summary, we find that all four-neutrino descriptions of the LSND anomaly both in $(2+2)$ as well as $(3+1)$ realizations are highly disfavoured due to recent data, as mentioned in our conclusions, Sec. 9. 


\section{Four-neutrino oscillations}

In this letter we interpret current neutrino oscillation data in a four-neutrino framework. This requires the existence of a fourth light neutrino, which must be sterile to account for the LEP invisible $\mathrm{Z}$ width data [21, 22, 23, 24, 29]. The simplest theoretical models add just one such light $S U(2) \otimes U(1)$ singlet neutrino and ascribe its lightness either to an underlying protecting symmetry, such as lepton number [21, 22], or to the existence of extra dimensions [25, 26]. Symmetry breaking is required in order to generate solar and atmospheric oscillations, characterized by splittings which arise due to small tree-level [27] or radiative effects [21, 22]. Many other four-neutrino models have been considered 24, 29].

Quite generally, the four-neutrino charged current leptonic weak interaction is specified as by a rectangular $3 \times 4$ lepton mixing matrix $K=\Omega U$, with $\Omega$ diagonalizing the $3 \times 3$ charged lepton mass matrix and $U$ diagonalizing the $4 \times 4$ Majorana neutrino mass matrix. Altogether the elements of the mixing matrix $K$ are characterized by 6 mixing angles and 3 physical phases [39, which could lead to $\mathrm{CP}$ violation in oscillation phenomena [40]. For simplicity we neglect $\mathrm{CP}$-violating phases, whose effects are expected to be small in the experiments we consider, due to the strong hierarchy of the mass-squared differences required by the experimental data. This leaves us with nine parameters altogether relevant for the description of CP conserving four-neutrino oscillations: 6 mixing angles contained in the mixing matrix and 3 mass-squared differences.

As shown in Ref. [28] it is very important to adopt a convenient choice for these parameters in order to factorize the analysis. Here we adopt all the notation and conventions introduced in Ref. [28], which are based on the use of physically relevant quantities. The 6 parameters $\Delta m_{\mathrm{SOL}}^{2}, \theta_{\mathrm{SOL}}, \Delta m_{\mathrm{ATM}}^{2}, \theta_{\mathrm{ATM}}, \Delta m_{\mathrm{LSND}}^{2}$, $\theta_{\mathrm{LSND}}$ are similar to the 2-neutrino mass-squared differences and mixing angles and are directly related to the oscillations in solar, atmospheric and the LSND experiments. For the remaining 3 parameters we use $\eta_{s}, \eta_{e}$ and $d_{\mu}$. Here, $\eta_{s}\left(\eta_{e}\right)$ is the fraction of $\nu_{s}\left(\nu_{e}\right)$ participating in solar oscillations, and $1-d_{\mu}$ is the fraction of $\nu_{\mu}$ participating in atmospheric oscillations (for exact definitions see Ref. [28]). For the analysis we adopt the following approximations:

- We make use of the hierarchy $\Delta m_{\mathrm{SOL}}^{2} \ll \Delta m_{\mathrm{ATM}}^{2} \ll \Delta m_{\mathrm{LSND}}^{2}$. This means that for each data set we consider only one mass-squared difference, the other two are set either to zero or to infinity.

- In the analyses of solar and atmospheric data (but not for SBL data) we set $\eta_{e}=1$, which is justified because of strong constraints from reactor experiments [14, 15, 16]. 


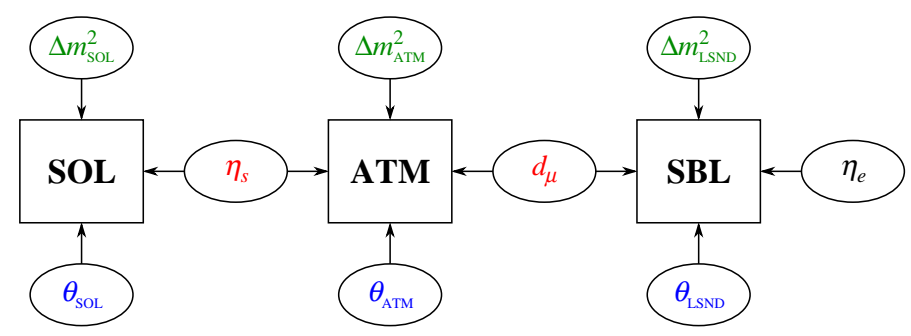

Figure 2. Parameter dependence of the different data sets in our parameterization.

Due to these approximations (well justified by reactor data and in oder to fit in LSND with solar + atmospheric data) the parameter structure of the problem gets rather simple [28]. The parameter dependence of the different data sets solar, atmospheric and SBL is illustrated in Fig. 2. We see that only $\eta_{s}$ links solar and atmospheric data and $d_{\mu}$ links atmospheric and SBL data. All the other parameters are "private" to one data set.

\section{Solar neutrinos}

Here we borrow from Ref. [4] a recent re-analysis of present solar data including a study of degree of rejection against the sterile neutrino oscillation hypothesis. The experimental data used are the solar neutrino rate of the chlorine experiment Homestake [6], the rates of the gallium experiments SAGE [7], GALLEX and GNO [8], as well as the 1496-day Super-Kamiokande data sample [5] in form of 44 recoil electron zenith-energy bins. Moreover, we include the data from the recent SNO charged current and neutral current event measurements, as well as day-night information in the form of 34 data points of the observed energy spectrum [10]. For the solar neutrino fluxes we use the Standard Solar Model fluxes [41], including its prediction of the ${ }^{8} \mathrm{~B}$ flux. The first hints in favour of the LMA solution [42], which followed mainly from the flatness of the Super-K spectrum, have now become a robust result, thanks to the new SNO data and additional Super-K spectrum data. In contrast with the pre-SNO-NC situation, presently all non-LMA solutions are strongly rejected, so that in this paper we will restrict the solar oscillation parameters to the LMA region.

Another important consequence of the recent SNO-NC data is specially relevant for us, namely, that the degree of rejection against solar neutrino oscillations to sterile neutrinos has now become much more significant. This results can be summarized in Fig. 3. The line labeled "after" shows $\Delta \chi_{\mathrm{SOL}}^{2}$ as a function of $0 \leq \eta_{s} \leq 1$, after recent solar neutrino up-dates, most important SNO-NC [10]. We minimize with respect to the other two solar neutrino oscillation parameters $\Delta m_{\mathrm{SOL}}^{2}$ and $\theta_{\mathrm{SOL}}$, required only to lie in the LMA region. For comparison we show also the line labeled "before", corresponding to the data 


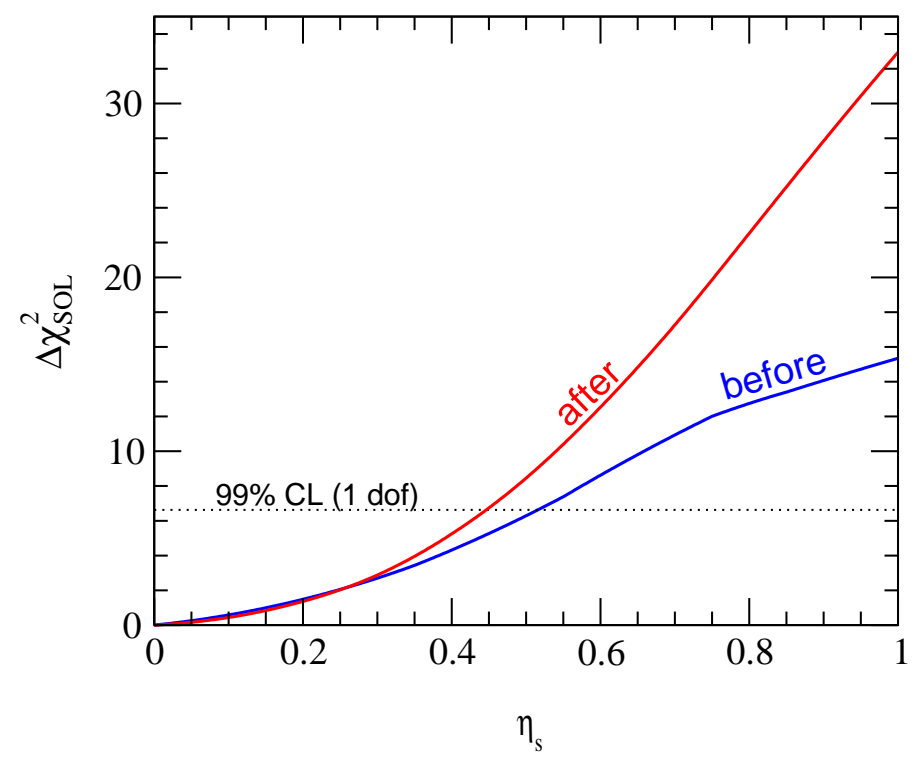

Figure 3. $\Delta \chi_{\mathrm{SOL}}^{2}$ for the favoured LMA solution, before and after SNO NC data [10], displayed as a function of $\eta_{s}$.

used in Ref. [28]. From this figure one can place a mass scheme-independent limit on the parameter $\eta_{s}$ from solar data:

$$
\text { solar data (LMA): } \eta_{s} \leq 0.45 \quad \text { (99\% C.L.), }
$$

compared to $\eta_{s} \leq 0.52$ obtained in Ref. [28]. Moreover, the pure sterile solution $\left(\eta_{s}=1\right)$ and large values of $\eta_{s}$ are now much more disfavoured than previously.

\section{Atmospheric neutrinos}

For the atmospheric data analysis [4] we use the following data from the SuperKamiokande experiment [2]: $e$-like and $\mu$-like samples of sub- and multi-GeV data, as well as the up-going stopping and through-going muon data. The Super-K data correspond to the 1489-day sample presented at Neutrino 2002 [2]. In contrast to previous analyses [28, 44, 45] here we adopt a more precise ten-bin presentation of the corresponding contained zenith-angle distributions. Further, we use the data from MACRO [3], including the recent update of their up-going muon sample (10 angular bins). Since $\Delta m_{\mathrm{SOL}}^{2} \ll \Delta m_{\mathrm{ATM}}^{2}$ we adopt the standard approximation of neglecting $\Delta m_{\mathrm{SOL}}^{2}$ when performing the analysis of the atmospheric data $\square$. For further details of the atmospheric neutrino analysis see Refs. 44, 45] and references therein.

Let us consider the impact of atmospheric data alone on the parameter $\eta_{s}$. As shown in Ref. [28] the behaviour of the atmospheric data as a function of

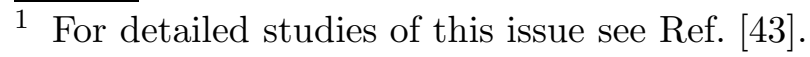



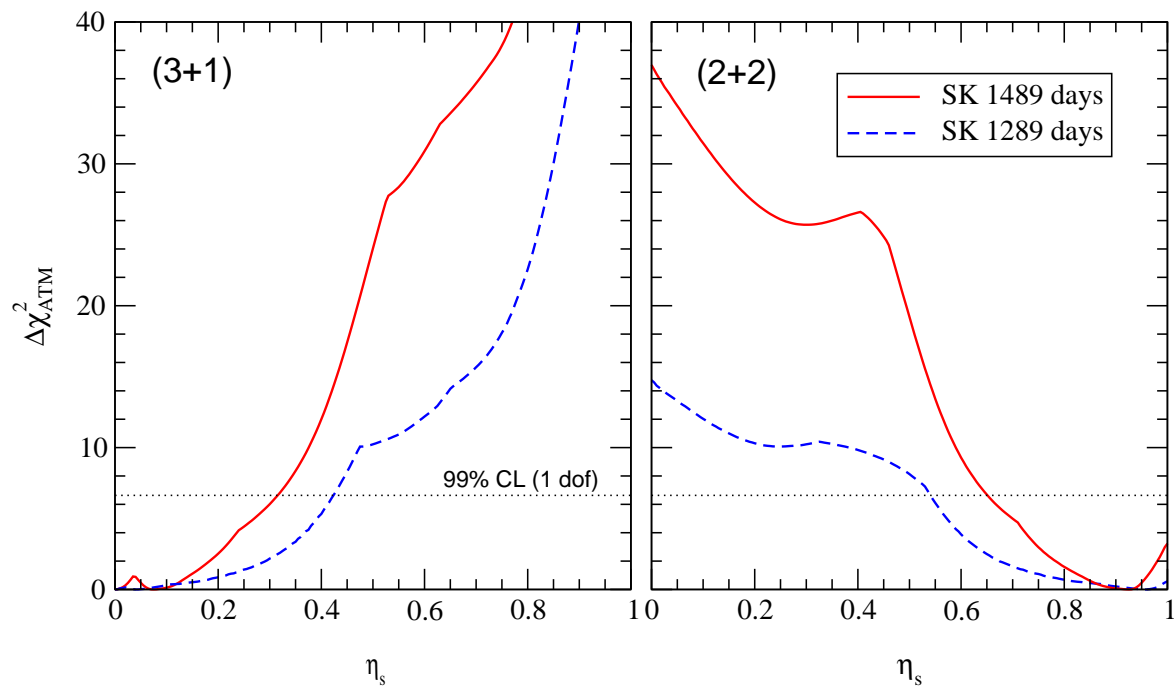

Figure 4. $\Delta \chi_{\text {ATM }}^{2}$ displayed as a function of $\eta_{s}$ for $(3+1)$ and $(2+2)$ schemes, before and after the most recent Super-K up-date [2].

$\eta_{s}$ depends on the mass schemes considered, with three inequivalent cases ${ }^{2}$ $(3+1)_{a, d},(3+1)_{b, c}$ and $(2+2)_{A, B}$. The qualitative behaviour of $(3+1)_{a, d}$ and $(3+1)_{b, c}$ schemes (see Fig. 1) is similar, however, small differences appear due to matter effects in atmospheric oscillations. In the following we will always minimize between these two cases and refer simply to the $(3+1)$ scheme.

We present in Fig. 4 the $\Delta \chi_{\text {ATM }}^{2}$ profile displayed as a function of $0 \leq \eta_{s} \leq 1$ minimized with respect to all other parameters, before and after the latest data of Ref. [2] have been included. We see that the new data significantly improve the sensitivity of atmospheric data with respect to a sterile component. Especially for $(2+2)$ schemes small values of $\eta_{s}$ are highly disfavoured, which will be crucial for the exclusion of $(2+2)$ spectra. From the figure we obtain the following bounds on $\eta_{s}$ at $99 \%$ C.L.:

$$
\text { atmospheric data: } \begin{cases}\eta_{s} \leq 0.32 & \text { for }(3+1) \text { schemes, } \\ \eta_{s} \geq 0.65 & \text { for }(2+2) \text { schemes. }\end{cases}
$$

\section{Reactor and accelerator data}

We divide the data from reactor and accelerator experiments in data from experiments reporting no evidence (NEV) for oscillations and in the LSND experiment. In this paper we compare the implications of two different analyses

$\overline{2}$ Note that $\eta_{s}$ is defined as the fraction of sterile neutrinos in solar oscillations and is only indirectly related to the fraction of sterile neutrinos in atmospheric oscillations. This relation is different for the various mass schemes [28]. 


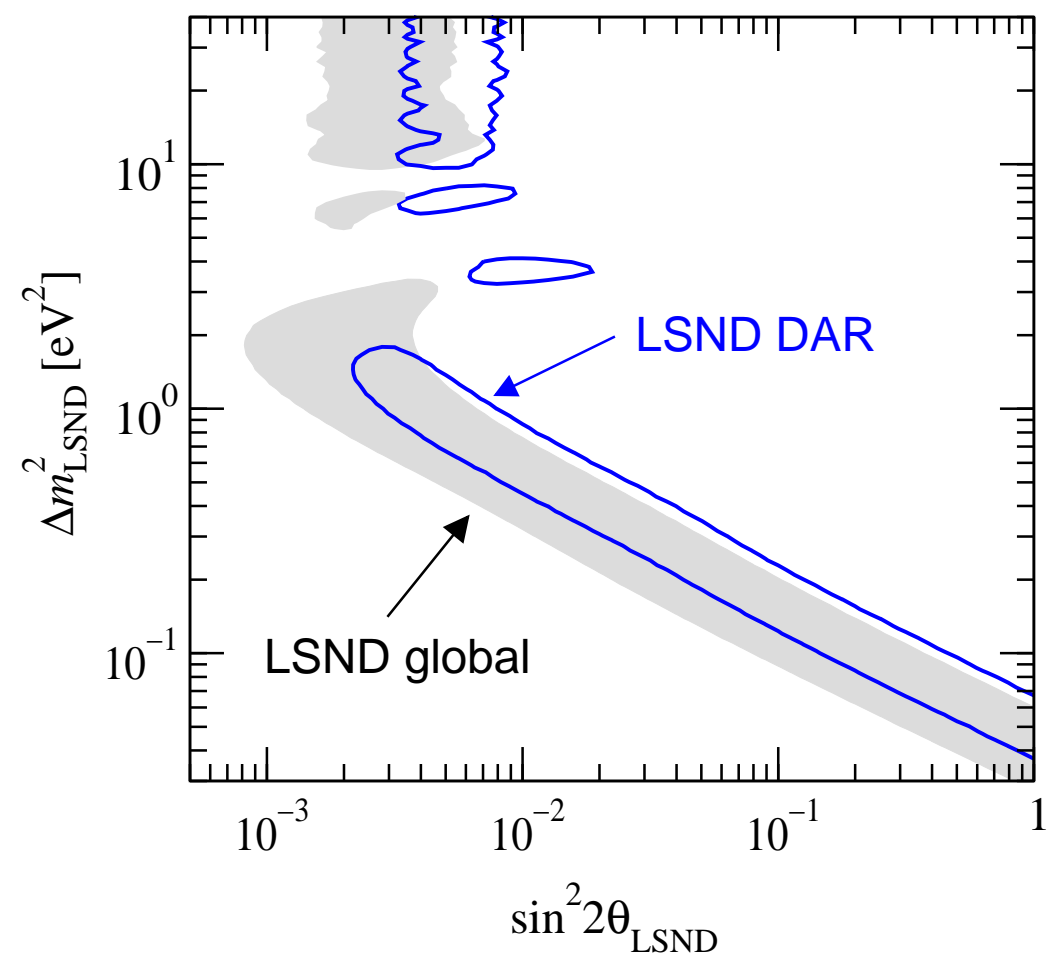

Figure 5. Comparison of the $99 \%$ C.L. regions of the global LSND analysis 19] (shaded region) and of the analysis performed in Ref. 31] (solid line) using mainly the decay-at-rest (DAR) data sample.

of the LSND data. First, we use the likelihood function obtained in the final LSND analysis [19] from their global data with an energy range of $20<$ $E_{e}<200 \mathrm{MeV}$ and no constraint on the likelihood ratio $R_{\gamma}$ (see Ref. [19] for details). This sample contains 5697 events including decay-at-rest (DAR) $\bar{\nu}_{\mu} \rightarrow \bar{\nu}_{e}$, and decay-in-flight (DIF) $\nu_{\mu} \rightarrow \nu_{e}$ data. We refer to this analysis as LSND global. Second, we use the LSND analysis performed in Ref. [31] based on 1032 events obtained from the energy range $20<E_{e}<60 \mathrm{MeV}$ and applying a cut of $R_{\gamma}>10^{-5}$. These cuts eliminate most of the DIF events from the sample, leaving mainly the DAR data, which are more sensitive to the oscillation signal. We refer to this analysis as LSND DAR.

In both cases the collaboration has provided us with the likelihood function obtained in their analyses [46, 47] which we convert into a $\chi^{2}$ according to $\chi^{2} \propto$ $-2 \ln \mathcal{L}$ (see Ref. [28] for details). In Fig. 5 we compare the $99 \%$ C.L. regions obtained from the two LSND analyses. The LSND DAR data prefers somewhat larger mixing angles, which will lead to a stronger disagreement of the data in $(3+1)$ oscillation schemes. Furthermore, the differences in $\chi^{2}$ between the best fit point and no oscillations for the two analyses are given by $\Delta \chi_{\text {no osc }}^{2}=29$ (global) and $\Delta \chi_{\text {no osc }}^{2}=47$ (DAR). This shows that the information leading to the positive oscillation signal seems to be more condensed in the DAR data.

Concerning the NEV data, we take into account the detailed information from 
the SBL disappearance experiments Bugey [14] and CDHS [17], as described in Ref. [36]. The constraints from KARMEN [13] are included by means of the likelihood function provided by the collaboration [47. Further, we include the results on the $\bar{\nu}_{e}$ survival probability from the long-baseline experiments CHOOZ [15] $(P=1.01 \pm 0.028 \pm 0.027)$ and Palo Verde [16] $(P=1.01 \pm$ $0.024 \pm 0.053)$ as explained in Ref. [28].

\section{$6(2+2)$ oscillations: ruled out by solar and atmospheric data}

The strong preference of oscillations into active neutrinos in solar and atmospheric oscillations leads to a direct conflict in $(2+2)$ oscillation schemes [28, 34, 45]. This is evident from the comparison of Eqs. (1) and (2). We will now show that thanks to the new SNO solar neutrino data 10 and the improved SK statistic on atmospheric neutrinos [2] the tension in the data has become so strong that $(2+2)$ oscillation schemes are, in contrast to Ref. [28], essentially ruled out.

In Fig. 6 we show the $\chi^{2}$ as a function of $\eta_{s}$ for solar data and for atmospheric combined with SBL data 3 . Furthermore, we show the $\chi^{2}$ of the global data defined by

$$
\bar{\chi}^{2}\left(\eta_{s}\right) \equiv \Delta \chi_{\mathrm{SOL}}^{2}\left(\eta_{s}\right)+\Delta \chi_{\mathrm{ATM}+\mathrm{SBL}}^{2}\left(\eta_{s}\right)
$$

In this section we investigate quantitatively the degree of disagreement of the two data sets solar and atmospheric+SBL in $(2+2)$ oscillation schemes using the statistical methods described in detail in the appendix.

First, in the method which we are calling parameter consistency test (PC) we calculate the C.L. at which still some value of the common parameter $\eta_{s}$ is allowed by both data sets. From Fig. 6 we find

$$
\chi_{\mathrm{PC}}^{2}=12.2 \text {. }
$$

This implies that only if we take both data sets at the $99.95 \%$ C.L. a value of $\eta_{s}$ exists, which is contained in the allowed regions of both sets.

Second, the parameter goodness of fit (PG) makes use of the $\bar{\chi}^{2}$ defined in Eq. (3). This criterion evaluates the g.o.f. of the combination of data sets, without being diluted by a large number of data points, as it happens for

3 For completeness we use here (atmospheric+NEV+LSND global) data. However, the impact of SBL data on the $\chi^{2}$-dependence on $\eta_{s}$ is very small, as can be seen by comparing the corresponding lines in Figs. 6 and 4. Also the differences between LSND global and LSND DAR are negligible in this case. 


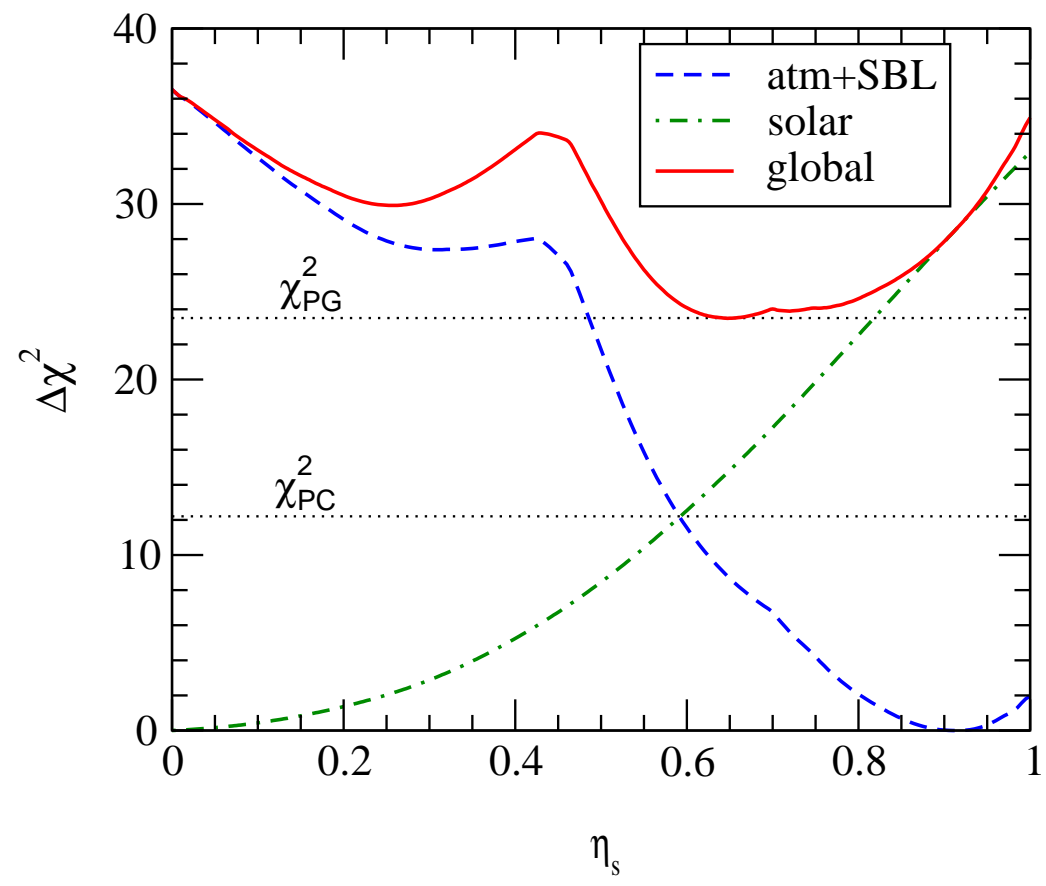

Figure 6. $\Delta \chi_{\mathrm{SOL}}^{2}, \Delta \chi_{\mathrm{ATM}+\mathrm{SBL}}^{2}$ and $\bar{\chi}_{\text {global }}^{2}$ as a function of $\eta_{s}$ in $(2+2)$ oscillation schemes. Also shown are the values $\chi_{\mathrm{PC}}^{2}$ and $\chi_{\mathrm{PG}}^{2}$ relevant for parameter consistency and parameter g.o.f., respectively.

the usual g.o.f. criterion. For the case of two data sets constraining one single common parameter this method is equivalent to subtracting the best fit values for the parameter obtained from the two data sets and calculating the C.L. at which the difference is consistent with zero. According to Eq. (A.5) we find

$$
\chi_{\mathrm{PG}}^{2} \equiv \bar{\chi}_{\min }^{2}=23.5
$$

Evaluated for 1 d.o.f. 5 such a high $\chi^{2}$-value leads to the marginal parameter g.o.f. of $1.3 \times 10^{-6}$.

From Eqs. (4) and (5) we conclude that $(2+2)$ oscillation schemes are highly disfavoured, if not ruled out: solar data and atmospheric+SBL data are in disagreement at the $3.5 \sigma$ level according to the PC test and at the $4.8 \sigma$ level according to the PG. This is a very robust result, independent of whether LSND is confirmed or disproved. We note, however, that in the unlikely event that the KamLAND reactor experiment 48 will see no signal and rule out the LMA solution, the fit of $(2+2)$ schemes may improve. In that case, although the solar fit quality will substantially deteriorate since we are left with non-LMA solar solutions, the agreement with atmospheric data will improve, especially for the quasi-vacuum case [4].

$\overline{4}$ Applying Eq. (A.5) we find for the number of d.o.f. $3_{\mathrm{SOL}}+7_{\mathrm{ATM}+\mathrm{SBL}}-9=1$ corresponding to the single common parameter $\eta_{s}$. 


\section{7 (3+1) oscillations: strongly disfavoured by SBL data}

It is known for a long time that $(3+1)$ mass schemes are disfavoured by the comparison of SBL disappearance data with the LSND result [32, 33]. Here we extend the first quantitative analyses of Refs. [36, 37] by evaluating whether the two LSND data sets (global [19] and DAR [31]) are consistent with all the rest of the global sample of oscillation data (SOL+ATM+NEV) by using the statistical methods explained in detail in the appendix.

Following previous works [36, 37] and the original analyses of Bugey [14] and CDHS [17] we perform the analysis for fixed values of $\Delta m_{\mathrm{LSND}}^{2}$. Therefore, the two data sets we are comparing depend on $1_{\mathrm{LSND}}$ and $8_{\mathrm{SOL}+\mathrm{ATM}+\mathrm{NEV}}$ parameters and have only the single parameter $\theta_{\mathrm{LSND}}$ in common. For each value of $\Delta m_{\mathrm{LSND}}^{2}$ the analysis is completely analogous to the one of the previous section. In Fig. 7 we show the results of the PC test and the PG test for both LSND data sets as a function of $\Delta m_{\mathrm{LSND}}^{2}$. The horizontal lines indicate the C.L. of $95.45 \%, 99.73 \%$ and $99.9937 \%$, corresponding to 2,3 and 4 standard deviations, respectively. From this figure we observe that the disagreement is roughly one standard deviation worse with the LSND DAR analysis compared to the LSND global data analysis. The larger values of $\sin ^{2} 2 \theta_{\text {LSND }}$ preferred by the DAR data are in stronger disagreement with the bounds of the NEV experiments.

The upper panel of Fig. 7 shows that for the global LSND data a value of $\theta_{\mathrm{LSND}}$ consistent with the rest of the oscillation data exists at best at the $2 \sigma$ level. For LSND DAR parameter consistency is possible only at a C.L. greater than $99 \%$ (for most $\Delta m_{\mathrm{LSND}}^{2}$ values, only at more than $3 \sigma$ ). The parameter g.o.f. is shown in the lower panel. For the global LSND data it is always worse than $3 \sigma$, except for $\Delta m_{\mathrm{LSND}}^{2} \sim 6 \mathrm{eV}^{2}$ it reaches $1 \%$. For LSND DAR it is for most values of $\Delta m_{\mathrm{LSND}}^{2}$ below $0.01 \%$. The weakest disagreement in all cases occurs for the "LSND island" around $\Delta m_{\mathrm{LSND}}^{2} \sim 6 \mathrm{eV}^{2}$. Note that this does not correspond to the best fit value in a global fit (see next section). For the LSND global data the best fit point is at $\Delta m_{\mathrm{LSND}}^{2}=27 \mathrm{eV}^{2}$ with a $\mathrm{PC}$ at $97 \%$ C.L. and a PG $0.2 \%$. For LSND DAR the best fit point is at $\Delta m_{\mathrm{LSND}}^{2}=0.91$ $\mathrm{eV}^{2}$ with a $\mathrm{PC}$ at $3 \sigma$ and an $\mathrm{PG}$ of $3 \times 10^{-5}$.

5 Here the NEV data plays a key role, but also atmospheric data is important in the lower regions of $\Delta m_{\mathrm{LSND}}^{2}$ [33, 37]. For completeness we include also solar data, although the impact on the LSND amplitude is very small. 


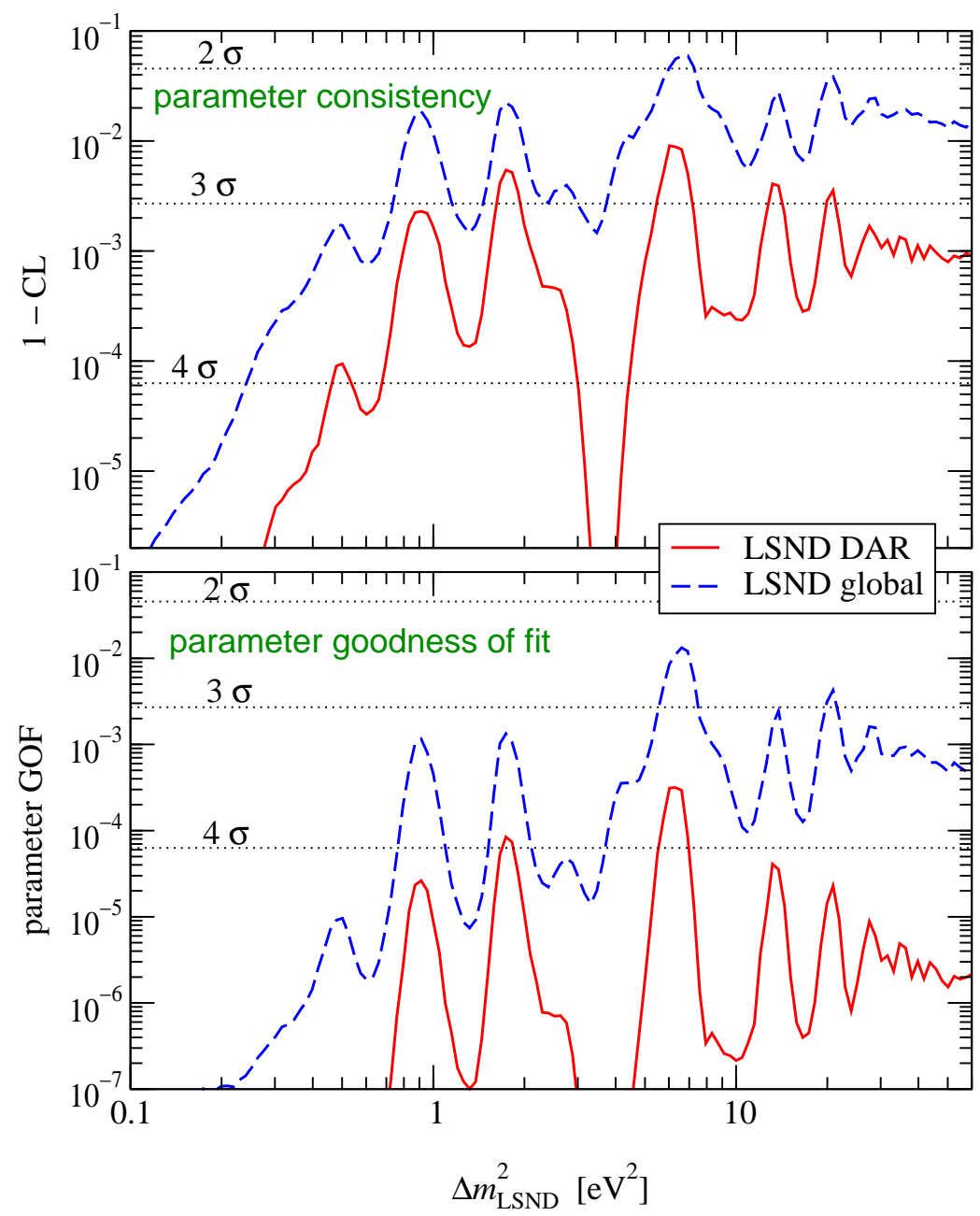

Figure 7. Compatibility of LSND with solar+atmospheric+NEV data in $(3+1)$ schemes. In the upper panel we show the C.L. of the parameter consistency whereas in the lower panel we show the parameter g.o.f. for fixed values of $\Delta m_{\mathrm{LSND}}^{2}$. The analysis is performed both for the global [19 and for the DAR 31 LSND data samples.

\section{Comparing (3+1), $(2+2)$ and (3+0) hypotheses}

With the methods developed in Ref. [28] we are able to perform a global fit to the oscillation data in the four-neutrino framework. This approach allows to statistically compare the different hypotheses. Let us first evaluate the g.o.f. of $(3+1)$ and $(2+2)$ spectra with the help of the PG method described in the appendix. We divide the global oscillation data in the 4 data sets SOL, ATM, NEV and LSND. According to Eq. (A.5) we consider the $\chi^{2}$-function

$$
\begin{aligned}
\bar{\chi}^{2} & =\Delta \chi_{\mathrm{SOL}}^{2}\left(\theta_{\mathrm{SOL}}, \Delta m_{\mathrm{SOL}}^{2}, \eta_{s}\right)+\Delta \chi_{\mathrm{ATM}}^{2}\left(\theta_{\mathrm{ATM}}, \Delta m_{\mathrm{ATM}}^{2}, \eta_{s}, d_{\mu}\right) \\
& +\Delta \chi_{\mathrm{NEV}}^{2}\left(\theta_{\mathrm{LSND}}, \Delta m_{\mathrm{LSND}}^{2}, d_{\mu}, \eta_{e}\right)+\Delta \chi_{\mathrm{LSND}}^{2}\left(\theta_{\mathrm{LSND}}, \Delta m_{\mathrm{LSND}}^{2}\right),
\end{aligned}
$$




\begin{tabular}{|l|cc|cc|}
\hline \hline & \multicolumn{2}{|c|}{ LSND global } & \multicolumn{2}{c|}{ LSND DAR } \\
\hline & $(3+1)$ & $(2+2)$ & $(3+1)$ & $(2+2)$ \\
\hline SOL & 0.0 & 14.8 & 0.0 & 14.8 \\
ATM & 0.4 & 6.7 & 0.2 & 6.7 \\
NEV & 7.0 & 9.7 & 17.1 & 12.2 \\
LSND & 7.2 & 1.2 & 6.8 & 1.9 \\
\hline$\chi_{\mathrm{PG}}^{2}$ & 14.6 & 32.4 & 24.1 & 35.6 \\
\hline $\mathrm{PG}$ & $5.6 \times 10^{-3}$ & $1.6 \times 10^{-6}$ & $7.6 \times 10^{-5}$ & $3.5 \times 10^{-7}$ \\
\hline \hline
\end{tabular}

Table 1

$\chi_{\mathrm{PG}}^{2}$ for $(3+1)$ and $(2+2)$ oscillation schemes in the global analysis for the two LSND data sets global [19] and DAR [31]. In the last row the parameter g.o.f. is shown for 4 d.o.f..

and the relevant number of d.o.f. is given by

$$
3_{\mathrm{SOL}}+4_{\mathrm{ATM}}+4_{\mathrm{NEV}}+2_{\mathrm{LSND}}-9_{\text {parameters }}=4
$$

This corresponds to the 4 parameters $\eta_{s}, d_{\mu}, \theta_{\mathrm{LSND}}, \Delta m_{\mathrm{LSND}}^{2}$ describing the coupling of the different data sets.

The results of this analysis is shown in Tab. 1. We show the contributions of the 4 data sets to $\chi_{\mathrm{PG}}^{2} \equiv \bar{\chi}_{\text {min }}^{2}$ for $(3+1)$ and $(2+2)$ oscillation schemes and for both LSND analyses. As expected we observe that in $(3+1)$ schemes the main contribution comes from SBL data due to the tension between LSND and NEV data in these schemes. For $(2+2)$ oscillation schemes a large part of $\chi_{\mathrm{PG}}^{2}$ comes from solar and atmospheric data, however, also SBL data contributes significantly. This comes mainly from the tension between LSND and KARMEN [31], which does not depend on the mass scheme and, hence, also contributes in the case of $(2+2)$. Therefore, the values of $\chi_{\mathrm{PG}}^{2}$ given in Tab. 1 for $(2+2)$ schemes are higher than the one of Eq. (5), where the tension in SBL data is not included.

In the last row of Tab. 1 we show the PG for the various cases. The best g.o.f. is obtained with the global LSND data in the $(3+1)$ scheme. However, even in this best case the g.o.f. is only $0.56 \%$. It is fair to say that all other cases are essentially ruled out by the PG criterion. In general the fit is worse with the LSND DAR analysis. Due to the larger values of $\sin ^{2} 2 \theta_{\text {LSND }}$ preferred by this data the disagreement between LSND and NEV experiments is significantly stronger than with the global data. We observe that the fit gets worse, if one selects LSND data with higher sensitivity to the oscillation signal. This seems rather discouraging for the four-neutrino hypothesis as an interpretation of LSND. 
Table 2

\begin{tabular}{|l|cc|}
\hline \hline$\Delta \chi^{2}$ & $(2+2)$ & $(3+0)$ \\
\hline LSND global & 17.8 & 20.0 \\
LSND DAR & 11.5 & 28.5 \\
\hline \hline
\end{tabular}

$\Delta \chi^{2}$ of $(2+2)$ and $(3+0)$ oscillation schemes with respect to $(3+1)$ spectra for the two LSND data sets global [19] and DAR [31].

Although we have seen that none of the four-neutrino mass schemes can provide a reasonable good fit to the global oscillation data including LSND, it might be interesting to consider the relative status of the three hypotheses $(3+1),(2+2)$ and the three-active neutrino scenario $(3+0)$. This can be done by comparing the $\chi^{2}$ values of the best fit point (which is in the $(3+1)$ scheme) with the one corresponding to $(2+2)$ and $(3+0)$. In Tab. 2 we show the $\Delta \chi^{2}$ of $(2+2)$ and $(3+0)$ with respect to $(3+1)$ for LSND global and DAR data.

First we observe that for LSND global $(2+2)$ is strongly disfavoured with respect to $(3+1)$ with a $\Delta \chi^{2}=17.8$. The reason for the big change with respect to the value of $\Delta \chi^{2}=3.7$ found in Ref. [28] is the improved sensitivity of solar (SNO NC) and atmospheric (SK 1489-days) data to a sterile component. With this new data now $(3+1)$ schemes are clearly preferred over $(2+2)$ : for 4 d.o.f. a $\Delta \chi^{2}=17.8$ implies that $(2+2)$ is ruled out at $99.87 \%$ C.L.. Further we see from Tab. 2 that now $(2+2)$ is only slightly better than $(3+0)$ for LSND global. Using LSND DAR data $(2+2)$ is somewhat less disfavoured with respect to $(3+1)$. The reason for this is that for LSND DAR $(3+1)$ gets much worse compared to LSND global, whereas the fit of $(2+2)$ deteriorates only slightly (see Tab. 1). Hence, the difference becomes smaller. Still, $(3+1)$ is clearly preferred: for 4 d.o.f. a $\Delta \chi^{2}=11.5$ implies that $(2+2)$ is ruled out at $97.9 \%$ C.L. with respect to $(3+1)$. Of course, for LSND DAR $(3+0)$ is very strongly disfavoured with a $\Delta \chi^{2}=28.5$ because of the high significance of the oscillation signal in this data set, which cannot be accounted for in the $(3+0)$ case.

\section{Conclusions}

Prompted by the recent SNO neutral current result and recent atmospheric data, we have re-analyzed the global status of all current neutrino oscillation data in the framework of four-neutrino schemes, in a way similar to that of Ref. [28]. Our present update includes the global solar neutrino data, the most recent sample of atmospheric neutrino experiments, and a detailed treatment of experiments reporting no evidence (NEV) for oscillations (KARMEN, Bugey, CDHS as well as information from CHOOZ and Palo Verde). Besides the analysis of the global LSND data, we also perform an analysis based mainly 
on the decay-at-rest (DAR) data sample, which provides a higher sensitivity to the oscillation signal in LSND. We have identified the parameter consistency (PC) test and the parameter goodness of fit (PG) as useful statistical methods to evaluate the compatibility of different data sets in a global analysis.

We have found that in $(2+2)$ mass schemes recent solar and atmospheric data are compatible only at the $3.5 \sigma$ according to the $\mathrm{PC}$ test, and at $4.8 \sigma$ according to the PG method. In $(3+1)$ mass schemes the disagreement of LSND with the rest of the oscillation data has been evaluated for both LSND analyses as a function of $\Delta m_{\mathrm{LSND}}^{2}$. For the global LSND data PC is achieved only at $2 \sigma$ and the PG is below $1 \%$ for all values of $\Delta m_{\mathrm{LSND}}^{2}$. Using the LSND DAR sample the disagreement is always stronger: we find a PC only at 99\% C.L. and for most values of $\Delta m_{\mathrm{LSND}}^{2}$ the PG is worse than $4 \sigma$.

We have evaluated the g.o.f. of a global fit in terms of the PG by dividing the data in SOL, ATM, LSND and NEV samples. In the best case we find a PG of only $0.56 \%$. This value occurs for the $(3+1)$ mass scheme and the global LSND data. Using the LSND DAR analysis we get a PG of $7.6 \times 10^{-5}$ for $(3+1)$. For $(2+2)$ oscillation schemes the situation is worse: we find a very bad PG of $1.6 \times 10^{-6}$ for LSND global and $3.5 \times 10^{-7}$ for LSND DAR. Concerning the relative status of the hypotheses $(3+1),(2+2)$ and the threeactive neutrino case $(3+0)$ we find that for the LSND global data $(2+2)$ and also $(3+0)$ are strongly disfavoured with respect to $(3+1)$ with a $\Delta \chi^{2}=17.8$ and $\Delta \chi^{2}=20.0$, respectively. For LSND DAR we find for $(2+2)$ a $\Delta \chi^{2}=11.5$ relative to $(3+1)$, and the high significance of the oscillation signal condensed in the DAR sample leads to the huge value of $\Delta \chi^{2}=28.5$ of $(3+0)$ relative to $(3+1)$.

The exclusion of four-neutrino oscillation schemes of the (2+2)-type is based on the improved sensitivity of solar and atmospheric neutrino experiments to oscillations into a sterile neutrino, thanks to recent experimental data. This is a very robust result, independent of whether the LSND experiment is confirmed or disproved. The exclusion of $(3+1)$ schemes depends somehow on the used LSND data sampleø. Furthermore, it heavily relies on the results of negative SBL experiments, especially on the Bugey and CDHS disappearance experiments. Therefore, if LSND should be confirmed by MiniBooNE, it will be crucial to improve the experimental data on SBL $\stackrel{(-)}{\nu}$ and/or $\stackrel{(-)}{\nu})$ disappearance. If the signal in LSND should indeed stem from oscillations in a $(3+1)$ mass scheme the SBL $\stackrel{(-)}{\nu}$ and $\stackrel{(-)}{\nu}_{\mu}$ disappearance amplitude must be just on the edge of the sensitivity of current experiments.

6 We want to stress that, although $(3+1)$ models themselves are not ruled out, they do not offer an acceptable framework for a combined description of current oscillation data, if LSND is included. In contrast, any model of the (2+2)-type is not viable if the gap separating the two pairs of neutrino states is large compared to $\Delta m_{\mathrm{ATM}}^{2}$. 
In summary, the interpretation of the global neutrino oscillation data including LSND in terms of four-neutrino mass schemes - in either $(3+1)$ or $(2+2)$ realizations - is strongly disfavoured. In the best case we obtain a parameter g.o.f. of only $0.56 \%$. The analysis we have presented brings the LSND anomaly to a theoretically more puzzling status indeed. We want to note that also introducing more sterile neutrinos participating in the oscillations is unlikely to substantially improve the situation [34]. If LSND should be confirmed by the results of the MiniBooNE experiment the situation will become even more puzzling. Examples of more far-fetched alternative explanations are the possibility of lepton number violating muon decay [49] or large CPT violation in the neutrino sector [50]. Such scenarios will be crucially tested at the upcoming experiments MiniBooNE [20] and KamLAND [48].

\section{A Statistical compatibility of different data sets}

In the global analysis of oscillation data in the framework of four-neutrino schemes we are faced with the problem that for a given mass scheme some data are in conflict with each other. In this appendix we present two statistical methods to evaluate quantitatively whether such data sets are consistent with each other.

Suppose a theoretical model characterized by $P$ parameters $x=\left(x_{1}, \ldots, x_{P}\right)$ and $K$ statistically independent experiments (or data sets) described by the theory. Each of these experiments may consist of many data points; experiment $k$ observes $N_{k}$ data points. Furthermore, experiment $k$ may in general depend only on $P_{k} \leq P$ parameters $x^{k}$, which is a sub-set of all parameters $x$ of the full theory, leading to the $\chi^{2}$-function

$$
\chi_{k}^{2}\left(x^{k}\right)=\left(\chi_{k}^{2}\right)_{\min }+\Delta \chi_{k}^{2}\left(x^{k}\right) .
$$

The three terms are distributed with $N_{k}, N_{k}-P_{k}, P_{k}$ degrees of freedom (d.o.f.). The global $\chi^{2}$ is given by

$$
\chi_{\text {tot }}^{2}(x)=\sum_{k=1}^{K} \chi_{k}^{2}\left(x^{k}\right)=\chi_{\min }^{2}+\Delta \chi^{2}(x) .
$$

Usually the goodness-of-fit (g.o.f.) is evaluated by considering the value of $\chi_{\text {min }}^{2}$, which is distributed with $N-P$ d.o.f., where $N=\sum_{k} N_{k}$.

Let us assume, that all the experiments considered separately lead to a good fit: $\left(\chi_{k}^{2}\right)_{\min } \approx N_{k}-P_{k}$. However, there may be some disagreement between different experiments. If the number of data points is very large the disagreement may be completely washed out and the usual g.o.f. would be very good, 
i.e. $\chi_{\min }^{2} \approx N-P$ despite the disagreement. $\square$ Here we propose two methods which are more useful in global analyses with many data points, like in the four-neutrino case.

\section{A.1 Parameter consistency test (PC)}

Let us consider the case of two data sets $(K=2)$, which have $\bar{P} \leq P$ parameters $\bar{x}$ in common. Then at a given C.L. the allowed regions for the parameters $\bar{x}$ according to each data set are determined by two $\chi^{2}$ functions $\Delta \chi_{1}^{2}(\bar{x})$ and $\Delta \chi_{2}^{2}(\bar{x})$, where we minimize with respect to the other parameters. Now we calculate $\chi_{\mathrm{PC}}^{2}$, defined as the minimal $\Delta \chi^{2}$ value where there is still some overlap between the allowed parameter regions of the two data sets. This $\chi_{\mathrm{PC}}^{2}$ has to be evaluated for $\bar{P}$ d.o.f. to obtain the C.L. at which there exists still some values of the parameters such that both data sets are consistent.

We call this criterion parameter consistency test. It can be applied if the global data can be divided into two potentially conflicting sets. Similar methods have been used in Refs. [36, 37] to evaluate the consistency of LSND and NEV experiments in $(3+1)$ schemes and in Ref. 31 to check the compatibility of LSND and KARMEN.

\section{A.2 Parameter goodness of fit $(P G)$}

Let us write the global $\chi^{2}$ Eq. (A.2) in the following way:

$$
\chi_{\text {tot }}^{2}(x)=\sum_{k=1}^{K}\left(\chi_{k}^{2}\right)_{\min }+\sum_{k=1}^{K} \Delta \chi_{k}^{2}\left(x^{k}\right) .
$$

To evaluate the g.o.f. we now use only the second term:

$$
\bar{\chi}^{2}(x) \equiv \sum_{k=1}^{K} \Delta \chi_{k}^{2}\left(x^{k}\right)=\bar{\chi}_{\min }^{2}+\Delta \bar{\chi}^{2}(x) .
$$

The first term in Eq. (A.3) describes the g.o.f. of all the experiments taken alone, whereas $\bar{\chi}_{\text {min }}^{2}$ in Eq. (A.4) corresponds to the contribution to the total minimum $\chi_{\min }^{2}$ which stems from the combination of the data sets. The three terms in Eq. (A.4) are distributed with $\sum_{k} P_{k}, \sum_{k} P_{k}-P, P$ d.o.f., respectively.

$\overline{7}$ For the global four-neutrino analysis this effect was discussed explicitly in Ref. [28]. 
Hence, to check the g.o.f. we just have to evaluate

$$
\chi_{\mathrm{PG}}^{2} \equiv \bar{\chi}_{\mathrm{min}}^{2} \quad \text { for } \quad \sum_{k=1}^{K} P_{k}-P \quad \text { d.o.f.. }
$$

We call this criterion parameter goodness of fit and in general it is more stringent than the parameter consistency test (for an illustration see Fig. 6).

If $M_{p} \leq K$ experiments depend on the parameter $x_{p}$ we have the obvious relation $\sum_{k=1}^{K} P_{k}=\sum_{p=1}^{P} M_{p}$, and the relevant number of d.o.f. given in Eq. (A.5) can be written as $\sum_{p=1}^{P} M_{p}-P=\sum_{p=1}^{P}\left(M_{p}-1\right)$. From this relation we see that parameters relevant for only one experiment $\left(M_{p}=1\right)$ do not contribute because they are minimized and contribute only to the individual $\left(\chi_{k}^{2}\right)_{\min }$ which are not considered in $\chi_{\mathrm{PG}}^{2}$. What is tested is only the parameter dependence of the data sets relevant for the combination.

A simple example: Let us consider the case of only one parameter $y$, which is determined by 2 experiments. Hence, we have $K=2$ and $P=P_{1}=P_{2}=1$ and $\chi_{\mathrm{PG}}^{2}=\min \left[\Delta \chi_{1}^{2}(y)+\Delta \chi_{2}^{2}(y)\right]$, which has to be evaluated for 1 d.o.f.. The following statement is easy to prove: Let $y_{1}$ and $y_{2}$ be the best fit values of the two experiments $\left(\Delta \chi_{k}^{2}\left(y_{k}\right)=0\right)$. Then the C.L. at which $y_{1}-y_{2}$ is consistent with zero is identical to the parameter goodness of fit.

\section{Acknowledgments}

We are grateful to W.C. Louis and G. Mills for providing us with the table of the global LSND likelihood function. Further, we thank K. Eitel for providing us with the likelihood functions of the LSND DAR and the KARMEN analyses and for very useful discussions. This work was supported by Spanish grant PB98-0693, by the European Commission RTN network HPRN-CT2000-00148 and by the European Science Foundation network grant N. 86. T.S. has been supported by the DOC fellowship of the Austrian Academy of Science and, in the early stages of this work, by a fellowship of the European Commission Research Training Site contract HPMT-2000-00124 of the host group. M.M. was supported by contract HPMF-CT-2000-01008 and M.A.T. was supported by the M.E.C.D. fellowship AP2000-1953.

\section{References}

[1] Y. Fukuda et al., Kamiokande Coll., Phys. Lett. B 335 (1994) 237; R. Becker-Szendy et al., IMB Coll., Nucl. Phys. B (Proc. Suppl.) 38 (1995) 331; W.W.M. Allison et al., Soudan Coll., Phys. Lett. B 449 (1999) 137. 
[2] M. Shiozawa, talk at Neutrino 2002, http://neutrino2002.ph.tum.de/; Super-Kamiokande Coll., Y. Fukuda et al., Phys. Rev. Lett. 81 (1998) 1562

[3] MACRO Coll., M. Ambrosio et al., Phys. Lett. B 434 (1998) 451; M. Spurio et al., hep-ex/0101019; A. Surdo, Talk given at TAUP 2001, 8-12 September 2001, Gran Sasso, Italy [http://www.lngs.infn.it/].

[4] M. Maltoni, T. Schwetz, M. A. Tórtola and J. W. F. Valle, hepph/0207227. For additional recent analyses of solar data see [51].

[5] M. Smy, talk at Neutrino 2002, http://neutrino2002.ph.tum.de/; S. Fukuda et al. [Super-Kamiokande Collaboration], Phys. Lett. B 539 (2002) 179 arXiv:hep-ex/0205075; Super-Kamiokande Coll., Y. Fukuda et al., Phys. Rev. Lett. 81, 1158 (1998); Erratum ibid. 81, 4279 (1998) and ibid. 82, 1810 and 2430 (1999); Y. Suzuki, Nucl. Phys. B (Proc. Suppl.) 77, 35 (1999); S. Fukuda et al., hep-ex/0103032.

[6] B.T. Cleveland et al., Astrophys. J. 496, 505 (1998); R. Davis, Prog. Part. Nucl. Phys. 32, 13 (1994); K. Lande, Talk given at Neutrino 2000, 15-21 June 2000, Sudbury, Canada [http://nu2000 . sno. laurentian.ca].

[7] SAGE Coll., D.N. Abdurashitov et al., Phys. Rev. C60, 055801 (1999); astro-ph/0204245.

[8] GALlEX Coll., W. Hampel et al., Phys. Lett. B447, 127 (1999); M. Altmann et al., GNO Coll., Phys. Lett. B 490 (2000) 16, hep-ex/0006034; C. Cattadori, GNO Coll., Nucl. Phys. B (Proc. Suppl.) 110 (2002) 311.

[9] Q. R. Ahmad et al. [SNO Collaboration], Phys. Rev. Lett. 87 (2001) 071301 nucl-ex/0106015.

[10] Q. R. Ahmad et al. [SNO Collaboration], Phys. Rev. Lett. 89, 011301 (2002) nucl-ex/0204008. Phys. Rev. Lett. 89, 011302 (2002) nucl$\mathrm{ex} / 0204009$.

[11] O. G. Miranda et al., Nucl. Phys. B 595 (2001) 360 hep-ph/0005259]. Phys. Lett. B 521 (2001) 299 hep-ph/0108145.

[12] M. Guzzo et al., Nucl. Phys. B 629 (2002) 479 [hep-ph/0112310].

[13] B. Armbruster et al., KARMEN Coll., Phys. Rev. D 65 (2002) 112001.

[14] B. Achkar et al., Bugey Coll., Nucl. Phys. B 434 (1995) 503.

[15] M. Apollonio et al., CHOOZ Coll., Phys. Lett. B 466 (1999) 415.

[16] F. Boehm et al., Palo Verde Coll., Phys. Rev. D 64 (2001) 112001 hep$\mathrm{ex} / 0107009$.

[17] F. Dydak et al., CDHS Coll., Phys. Lett. B 134 (1984) 281.

[18] C. Athanassopoulos et al., LSND Coll., Phys. Rev. Lett. 77 (1996) 3082; ibid 81 (1998) 1774.

[19] A. Aguilar et al. [LSND Collaboration], Phys. Rev. D 64 (2001) 112007 hep-ex/0104049.

[20] MiniBooNE Coll., R. Tayloe, Talk at Neutrino 2002, Munich, http://neutrino2002.ph.tum.de/.

[21] J. T. Peltoniemi, D. Tommasini and J. W. F. Valle, Phys. Lett. B 298 (1993) 383.

[22] J. T. Peltoniemi and J. W. F. Valle, Nucl. Phys. B 406, 409 (1993) hep- 
$\mathrm{ph} / 9302316]$.

[23] D.O. Caldwell and R.N. Mohapatra, Phys. Rev. D 48 (1993) 3259.

[24] Talks by E. Ma and by E.J. Chun, A. Joshipura and A. Smirnov, published in Elementary Particle Physics: Present and Future (World Scientific, 1996), ISBN 981-02-2554-7; E. Ma and P. Roy, Phys. Rev. D 52 (1995) R4780; J. J. Gomez-Cadenas and M.C. Gonzalez-Garcia, Z. Phys. C 71 (1996) 443; S. Goswami, Phys. Rev. D 55 (1997) 2931; Q.Y. Liu and A. Smirnov, Nucl. Phys. B 524 505; V. Barger, K. Whisnant and T. Weiler, Phys. Lett. B 427 (1998) 97; S. Gibbons, R.N. Mohapatra, S. Nandi and A. Raichoudhuri, Phys. Lett. B 430 (1998) 296; Nucl. Phys. B 524 (1998) 505; P. Langacker, Phys. Rev. D 58 (1998) 093017; M. Bando and K. Yoshioka, Prog. Theor. Phys. 100 (1998) 1239; W. Grimus, R. Pfeiffer and T. Schwetz, Eur. Phys. J. C 13 (2000) 125; F. Borzumati, K. Hamaguchi and T. Yanagida, Phys. Lett. B 497 (2001) 259; K. R. Balaji, A. Perez-Lorenzana and A. Y. Smirnov, Phys. Lett. B 509 (2001) 111; K.S. Babu and R.N. Mohapatra, hep-ph/0110243; S. Goswami and A.S. Joshipura, hep-ph/0110272.

[25] R. N. Mohapatra, A. Perez-Lorenzana and C. A. de S Pires, Phys. Lett. B 491 (2000) 14.

[26] A. Ioannisian and J. W. F. Valle, Phys. Rev. D 63 (2001) 073002; hepph/9911349.

[27] M. Hirsch and J. W. F. Valle, Phys. Lett. B 495 (2000) 121 hepph/0009066.

[28] M. Maltoni, T. Schwetz and J. W. Valle, Phys. Rev. D 65 (2002) 093004 hep-ph/0112103.

[29] Web-page of C. Giunti, http://www.to.infn.it/〜giunti/neutrino/ .

[30] V. Barger et al., Phys. Lett. B 489 (2000) 345.

[31] E. D. Church, K. Eitel, G. B. Mills and M. Steidl, Phys. Rev. D 66 (2002) 013001 hep-ex/0203023.

[32] S.M. Bilenky, C. Giunti and W. Grimus, in Proceedings of Neutrino '96, Helsinki, Finland, 13-19 June 1996, p. 174; Eur. Phys. J. C 1 (1998) 247; N. Okada and O. Yasuda, Int. J. Mod. Phys. A 12 (1997) 3669; V. Barger, et at., Phys. Rev. D 58 (1998) 093016.

[33] S.M. Bilenky, C. Giunti, W. Grimus and T. Schwetz, Phys. Rev. D 60 (1999) 073007.

[34] O.L.G. Peres and A.Yu. Smirnov, Nucl. Phys. B 599 (2001) 3.

[35] C. Giunti and M. Laveder, JHEP 0102 (2001) 001.

[36] W. Grimus and T. Schwetz, Eur. Phys. J. C 20 (2001) 1 hep-ph/0102252.

[37] M. Maltoni, T. Schwetz and J. W. F. Valle, Phys. Lett. B 518, 252 (2001) hep-ph/0107150.

[38] P. Di Bari, Phys. Rev. D 65 (2002) 043509; S.H. Hansen, et al., Phys. Rev. D 65 (2002) 023511; K.N. Abazajian, astro-ph/0205238.

[39] J. Schechter and J. W. F. Valle, Phys. Rev. D 22 (1980) 2227.

[40] J. Schechter and J. W. F. Valle, Phys. Rev. D 23 (1981) 1666.

[41] Web-page of J.N. Bahcall, http://www.sns.ias.edu/ jnb/ and refer- 
ences therein.

[42] M. C. Gonzalez-Garcia, P. C. de Holanda, C. Pena-Garay and J. W. Valle, Nucl. Phys. B 573 (2000) 3 [hep-ph/9906469].

[43] M. C. Gonzalez-Garcia and M. Maltoni, hep-ph/0202218.

[44] M. C. Gonzalez-Garcia, M. Maltoni, C. Pena-Garay and J. W. F. Valle, Phys. Rev. D 63 (2001) 033005 hep-ph/0009350; N. Fornengo, M.C. Gonzalez-Garcia and J. W. F. Valle, Nucl. Phys. B 580 (2000) 58; M. C. Gonzalez-Garcia, H. Nunokawa, O. L. G. Peres and J. W. F. Valle, Nucl. Phys. B 543 (1999) 3 [hep-ph/9807305].

[45] M.C. Gonzalez-Garcia, M. Maltoni and C. Peña-Garay, Phys. Rev. D 64 (2001) 093001 hep-ph/0105269 and hep-ph/0108073.

[46] W.C. Louis and G. Mills, LSND Coll., private communication.

[47] K. Eitel, KARMEN Coll., private communication.

[48] A. Piepke, KamLAND Coll., Nucl. Phys. B (Proc. Suppl.) 91, 99 (2001); http: //kamland.lbl.gov/KamLAND.

[49] K. S. Babu and S. Pakvasa, hep-ph/0204236.

[50] H. Murayama and T. Yanagida, hep-ph/0010178; G. Barenboim et al., hep-ph/0108199; A. Strumia, hep-ph/0201134.

[51] J.N. Bahcall et al., hep-ph/0204314; A. Bandyopadhyay et al., hepph/0204286; V. Barger et al., hep-ph/0204253; P.C. de Holanda, A.Yu. Smirnov hep-ph/0205241 G. L. Fogli et al., hep-ph/0206162; A. Strumia et al., hep-ph/0205261. 